\title{
Special Issue in Honor of Dr. Dianna Johnson
}

\author{
Eldon E. Geisert
}

Published online: 18 February 2011

(C) Springer Science+Business Media, LLC 2011

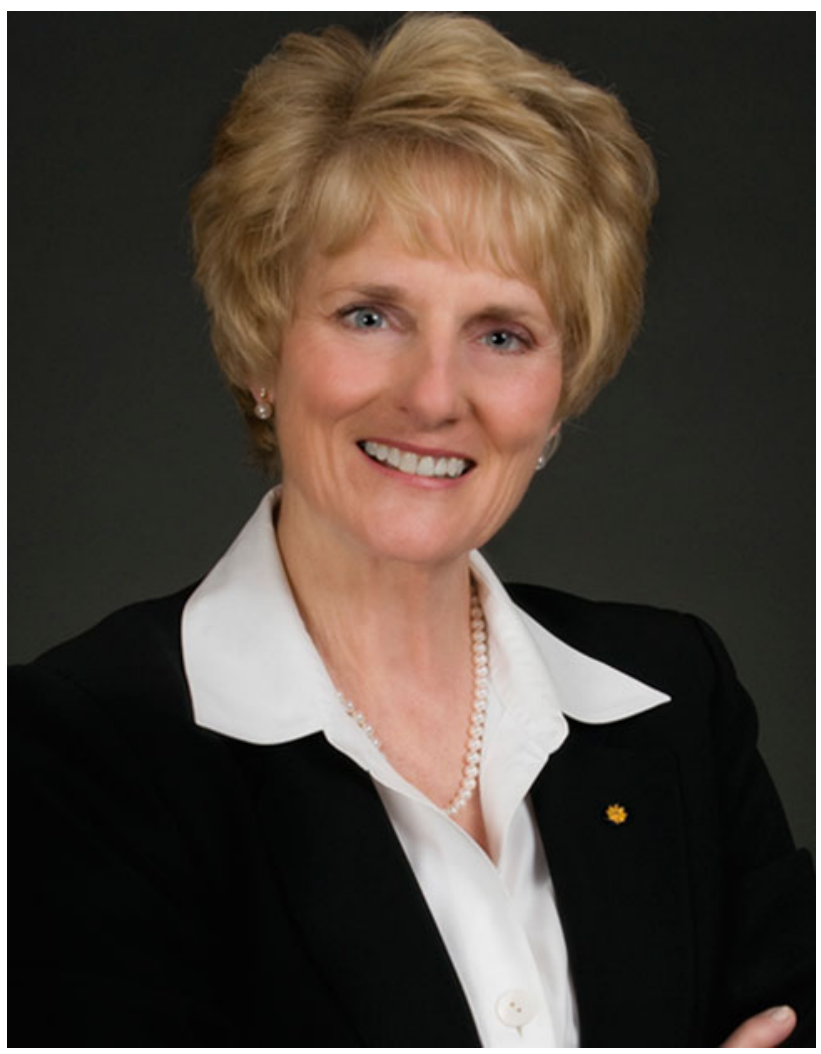

Dr. Dianna Johnson

It is a privilege to be the guest editor of the special issue honoring Dr. Dianna (Redburn) Johnson. Dr. Johnson has a long and exemplary career, serving as a role model for many young scientists. Dianna's entre to neuroscience

E. E. Geisert $(\bowtie)$

University of Tennessee, HSC, Memphis, TN, USA

e-mail: egeisert@uthsc.edu began in 1967 with a chance interview with Dr. Fred Samson, Chair of the Department of Physiology and Cell Biology at the University of Kansas, who had just returned the previous month from a sabbatical at UCLA to learn more about the emerging discipline of neurochemistry. Caught up in his fresh enthusiasm and a new appreciation of the complexities of the chemistry of the brain, Dianna signed up as his graduate student to study microtubules, 
which were speculated to serve axons not only as a cytoskeleton but also as a transport system for delivery of neurotransmitter vesicles to axon terminals. Early studies were multidisciplinary and collaborative within a large and interactive lab group, involving electron microscopy (using the newly discovered fixative, glutaraldehyde that for the first time allowed preservation of microtubule structure), creation and characterization of antibodies against microtubule protein, establishing rates of microtubule turnover and axonal transport. From this early point in her career, she was imprinted with the power and logic of collaborative research. Dianna joined the laboratory of Dr. Carl Cotman for a postdoctoral fellowship in the Department of Psychobiology at U.C. Irvine. I was an undergraduate in Dr. Cotman's laboratory, and have had the good fortune of interacting with Dianna ever since. In Dr. Cotman's laboratory, Dianna's experiences reinforced the power of collaborative research, providing a rich, highly interactive environment for further studies of the role of microtubules in synaptic transmission, as well as an intense introduction to techniques of subcellular fractionation. This rather brutal treatment of delicate neuronal tissue could, under favorable conditions, produce enriched, viable fractions of nerve endings that were pinched off and resealed: synaptosomes. These could now be used for functional studies in vitro. No longer did the business ends of the axon have to remain hidden in the complexities of the neuropile; you could hold them in your hand (test tube) and probe them directly for answers about neurotransmission. Armed with a custom fabricated instrument of her own design, Dianna determined the characteristics of synaptosomal neurotransmitter release convincing enough for a publication in Science.

Dianna was fortunate to join a new and thriving neuroscience group within a recently established medical school in Houston as an Assistant Professor. Helping to build a new Department of Neurobiology and Anatomy and forging her own research program made for heady times. She made a conscious decision to try and target a tractable part of the brain. The neural retina seemed to fit the bill, since it contained highly laminar structure and some of the largest synaptic endings in the CNS-what an opportunity for synaptosomes! Essentially her life's work in research has been to examine the exquisite synaptic architecture of the retina, its neurotransmitter connections and the remarkable orchestration that leads to its development. In the newly formed Department of Neurobiology and Anatomy she was given the opportunity to not only grow her own research, but to delve into administrative matters by helping to develop training programs for the Department and the Medical School and to create large collaborative working groups into research centers focused on visual neuroscience. In that environment she was able to compete successfully not only for individual R01 research grant funding, but also to add to programmatic funding that included training grants, shared facilities grants and substantial foundation and private funding.

Recruitment to the University of Tennessee Health Science Center in 1996 provided a chance to again help build a collaborative research group and to replicate some of the lessons learned in Houston. Under the strong leadership of Dr. Barrett Haik, the Department experienced stunning successes in recruiting new faculty and creating new laboratories that now constitute the Hamilton Eye Institute Vision Research Center. In this process, Dianna was instrumental in garnering shared core facility grants, obtaining grants from NEI as well as important private foundation and individual philanthropic support. Dianna also has concurrently pursued her passion for helping train the next generation of researchers and to work with the University to establish mentored research training programs for postdoctoral fellows and junior faculty. She has expanded her own research to include an interest in retinal development that led to the discovery of the early life of neurotransmitters as developmental factors that orchestrate synaptic connectivity. Recent collaborations with Dr. Michael Dyer have led to an expanded appreciation of synaptic differentiation in neuronal tumors, the expression of neurotransmitter agents in cancer and the possibility of using transmitter chemistry to direct drug development for treatment of ocular tumors. Thank you Dianna for your welcoming manner, your friendship and your wholehearted commitment to the many lives you have touched. 